\title{
Parents with unilateral retinoblastoma: their affected children
}

Corey M Notis, Kevork Niksarli, David H Abramson, Anthony R DeLillo, Robert M Ellsworth

\begin{abstract}
Background-A retrospective analysis of all patients with retinoblastoma on file at the Ophthalmic Oncology Center of the New York Hospital, Cornell University Medical Center was performed to examine the characteristics of retinoblastoma in children with a unilaterally affected parent. Methods-Data were analysed for laterality of disease, age at initial diagnosis, number of tumour foci at diagnosis, ocular outcome, and patient survival.

Results-There were 54 patients, including nine pairs of siblings, diagnosed with retinoblastoma, each of whom had one unilaterally affected parent. The retinoblastoma was bilateral in $49(91 \%)$ patients and unilateral in the remaining five $(9 \%)$. The mean age at diagnosis was 10.5 months (range 1-36 months) and 35 patients $(65 \%)$ were diagnosed within the first year of life. The mean number of initial tumour foci was 1.7 per eye; 1.6 foci per eye in bilaterally affected patients, and 3.7 foci per eye in unilaterally affected patients $(p=0 \cdot 36)$. Retention of the eye was possible for 51 of 103 eyes affected with retinoblastoma. The mean follow up for patients was 9.0 years, with a range of 1 month to 30.5 years. There were a total of 12 deaths (22\%): from retinoblastoma (two), sarcoma (five), cutaneous malignant melanoma (one), cancer type unknown (two), and cause unknown (two). Conclusion-Overall, the characteristics of retinoblastoma patients with a unilaterally affected parent, were similar when compared with all retinoblastoma patients with a positive family history. (Br f Ophthalmol 1996; 80: 197-199)
\end{abstract}

Ophthalmic Oncology Center, New York Hospital, Cornell

University Medical

Center, New York,

USA

C M Notis

K Niksarli

D H Abramson

A R DeLillo

R M Ellsworth

Correspondence to: Corey M Notis, MD, Center, K811, 525 East 68th Center, K811, 525 East 10021, USA.

Accepted for publication 13 September 1995

Retinoblastoma is the most common primary malignant intraocular tumour of childhood. The frequency of retinoblastoma varies from 1:17000 to 1:34 000 live births, or approximately 350 new cases each year in the United States. ${ }^{1}$ Retinoblastoma is unilateral in approximately two thirds of the cases and is bilateral in the remaining one third. All children with bilateral disease carry the germinal mutation, and are therefore classified as heritable cases. In addition, approximately $8 \%$ of unilaterally affected patients carry the germinal mutation, and are also classified as heritable cases. ${ }^{2}$ Yet children born to parents with unilateral or bilateral disease are followed in identical examination schedules. While many studies have detailed the characteristics of children with a family history of parental retinoblastoma, ${ }^{2-4}$ we have no information about the laterality or timing of tumour development regarding children affected with retinoblastoma who have a unilaterally affected parent. This study examines these affected children whose parents were diagnosed with unilateral retinoblastoma.

\section{Methods}

A retrospective review of 1753 cases on file at the Ophthalmic Oncology Center of the New York Hospital, Cornell University Medical Center was performed. Patients chosen for the study were limited to children who were diagnosed with retinoblastoma (unilateral or bilateral), who had a parent diagnosed with unilateral retinoblastoma. There were no patients who had more than one affected parent. The following variables were analysed in these selected patients: laterality, age at diagnosis, number of tumour foci, ocular outcome, and patient survival.

\section{Results}

\section{INCIDENCE AND LATERALITY}

There were 54 retinoblastoma patients identified as having a parent with unilateral retinoblastoma. The retinoblastoma was bilateral in 49 patients $(91 \%)$ and unilateral in the remaining five patients (9\%), yielding 103 affected eyes.

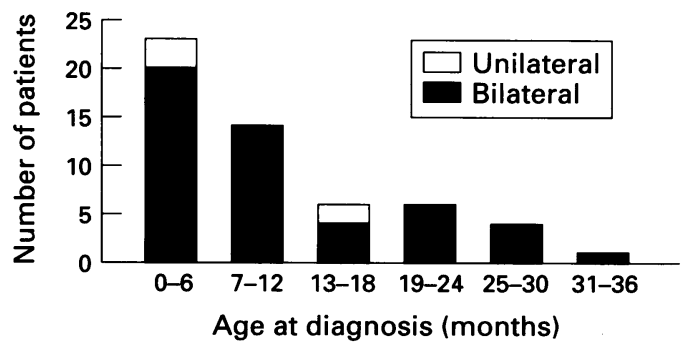

Figure 1 Age at initial diagnosis of retinoblastoma. 


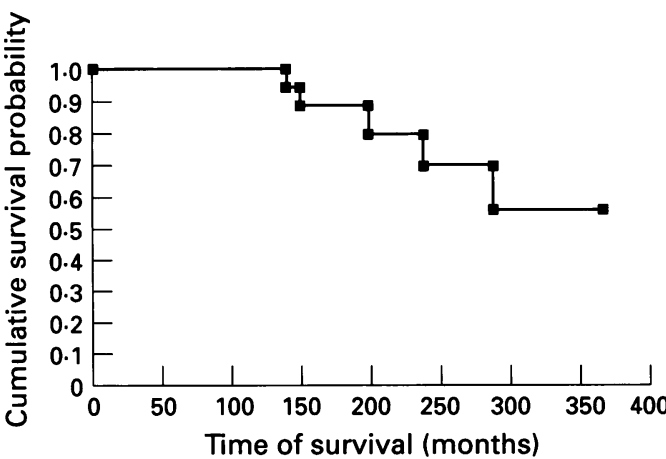

Figure 2 Survival of retinoblastoma patients.

\section{AGE AT DIAGNOSIS}

The average age at diagnosis of the children was 10.5 months with a range of $1-36$ months (Fig 1). Twenty three patients (43\%) were diagnosed within the first 6 months of life, and 37 patients $(69 \%)$ were diagnosed within the first year of life. Three of the five unilaterally affected patients were diagnosed within the first 6 months of life.

FAMILY HISTORY

All 54 patients in this study had only one affected parent. There were nine pairs of siblings included as well. The affected parent was the mother in 30 cases and the father in 24 cases.

\section{TUMOUR FOCI AT DIAGNOSIS}

The mean number of initial tumour foci was 1.7 per eye. There were 1.6 initial tumour foci per eye $(n=36)$ in patients with bilateral disease, compared with $3 \cdot 7$ foci per eye $(n=3)$ in patients with unilateral disease $(p=0.36)$. The number of foci could not be determined in 64 eyes; 39 eyes presented with massive tumour, and there were incomplete data regarding 25 eyes.

\section{OCULAR OUTCOME}

Retention of the eye was possible for 51 out of 103 eyes (50\%) affected with retinoblastoma. Of the 49 patients with bilateral disease, 14 $(29 \%)$ had bilateral enucleations, and 24 $(49 \%)$ had an enucleation of one eye. None of the unilaterally affected patients underwent an enucleation.

\section{PATIENT SURVIVAL}

The mean follow up for patients was $9 \cdot 0$ years, with a range of 1 month to 30.5 years (Fig 2). There were 12 deaths from the

Table 1 Mortality of patients with retinoblastoma

\begin{tabular}{lcc}
\hline Cause of death & $\begin{array}{l}\text { No of } \\
\text { patients }\end{array}$ & Age (years) \\
\hline Retinoblastoma & 2 & $1 \cdot 5,3 \cdot 8$ \\
Sarcoma & 5 & $11 \cdot 5,12 \cdot 5,17 \cdot 0,20 \cdot 0,24 \cdot 0$ \\
Malignant melanoma & 1 & $27 \cdot 5$ \\
Cancer - unknown type & 2 & $10 \cdot 0,19 \cdot 0$ \\
Unknown & 2 & $5 \cdot 5,13 \cdot 0$ \\
Total & 12 & \\
\hline
\end{tabular}

following causes (Table 1): two retinoblastoma, five sarcoma, one cutaneous malignant melanoma, two cancer (unknown site), and two unknown. Based on a life table analysis, overall patient survival at 30.5 years was $55 \%$.

\section{Conclusion}

All patients with bilateral retinoblastoma carry the germinal mutation, and are therefore heritable cases. Patients with unilateral retinoblastoma represent heritable cases in approximately $8 \%$ of cases. Since the retinoblastoma gene functions phenotypically in an autosomal dominant manner, half of the offspring of a parent with the germinal mutation will develop the disease. For children of a unilaterally affected patient this represents only a $4 \%$ risk.

There is currently no information regarding the laterality or timing of tumour development in children who have a unilaterally affected parent. Yet these children are followed in the same examination schedule as the children of a parent with bilateral disease. Subsequently, care of these children has always presented a unique challenge to the ophthalmic oncologist who must weigh the risk of tumour development against the risk from frequent examinations under anaesthesia. This dilemma has been aided by recent advances in the field of genetics, which has identified the retinoblastoma gene mutation through blood sample analysis. However, this test currently has its lowest yield for the children of parents with unilateral disease. In this study, the authors have examined the characteristics of retinoblastoma in children with unilaterally affected parents.

The presence of the germinal mutation in the unilaterally affected parent is confirmed by the high proportion of bilateral disease in the offspring $(91 \%)$, as well as the number of tumours per eye ( $1.7 \mathrm{foci})$. It is also evidenced by the presence of retinoblastoma among siblings. Interestingly, there are five cases present which appear to be heritable unilateral retinoblastoma.

The early age of diagnosis of retinoblastoma in the majority of cases is presumably due to the presence of family history of retinoblastoma leading to earlier, and more frequent, examinations. This concurs with the overall population of patients with a positive family history of retinoblastoma (whereby over $90 \%$ are diagnosed before 3 years of age), 5 and confirms the need for close and frequent follow up for all patients with a family history of retinoblastoma. While there were no diagnoses of retinoblastoma made after 3 years of age, given the small number of patients in this study, observation past 3 years of age is certainly advisable.

The ocular outcome and patient survival (including deaths from second tumours) was congruent with previous data concerning all patients with a germinal mutation. ${ }^{6}$ Despite the fact that a parent was affected, and that the disease was diagnosed at an early age, half of 
the eyes were not able to be salvaged, and the overall mortality was $22 \%$.

Overall, patients affected with retinoblastoma who had a unilaterally affected parent were similar when compared with all retinoblastoma patients with a positive family history. The majority of patients are affected with bilateral disease, and the mean age of diagnosis is very young, and there is usually more than one tumour per eye. There is an upper age limit (3 years) after which no new tumours are seen, but these children continue to be at risk for the development of second tumours. Until more sensitive genetic testing is widely available, frequent examination within the first 3 years of life is indicated for these patients.

1 Abramson DH. Retinoblastoma; diagnosis and management. Cancer F Clinicians 1982; 32: 130-40.

2 Wiggs JL, Dryja TP. Predicting the risk of hereditary retinoblastoma. Am $\mathscr{f}$ Ophthalmol 1988; 106: 346-51.

3 Gallie BL, Dunn JM, Hamel PA, Phillips RA. The genetics of retinoblastoma: relevance to the patient. Pediatr Clin N Am 1991; 38: 298-315.

4 Abramson DH, Ellsworth RM, Grumbach N, Kitchin FD. Retinoblastoma survival, age of detection and comparison. Retinoblastoma survival, age of detection and comparison. I Pediatr Ophthalmol Strabismus 1985; 22: 246-50.

Niksarli K, Abramson DH, Ellsworth RM, Servodidio CA. Changing trends in the management of retinoblastoma [Abstract]. Invest Ophthalmol Vis Sci 1993; 34: 1228.

6 Abramson DH, Ellsworth RM, Kitchin FD. Second monocular tumors in retinoblastoma survivors: are they radiation induced? Ophthalmology 1984; 91: 1351-5. 